CASE REPORT

\title{
Plantar Fibromatosis: An Unusual Cause of Plantar Pain
}

\author{
${ }^{1}$ Radiology Department, Braga Hospital, Portugal \\ ${ }^{2}$ Radiology Department, Clinical Diagnostic Imaging (CDPI), Brazil \\ ${ }^{3}$ Radiology Department, Federal Fluminense University, Brazil \\ ${ }^{4}$ Radiology Department, Federal University of Rio de Janeiro, Brazil
}

Filipa Vieira ${ }^{1 *}$, Clarissa Canella ${ }^{2,3}$, Flavia Costa ${ }^{2}$, Alessandro Severo ${ }^{3}$ and Edson Marchiori ${ }^{4}$

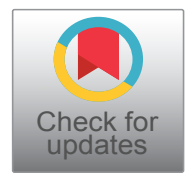

*Corresponding author: Filipa Vieira, Radiology Department, Braga Hospital, Portugal, Tel: +353913531207, E-mail:

f_g_v@msn.com

\begin{abstract}
Ledderhose's disease or plantar fibromatosis is a rare benign condition characterized by local proliferation of fibrous nodular tissue within the plantar aponeurosis. The diagnosis of this disease is based on clinical examination but imaging is often required to confirm the diagnosis or reveal alternative diagnosis. Because the cause of Ledderhose disease is still unknown, numerous therapeutic approaches for its treatment are available, from conservative to surgical. In this article, the authors describe case of a 46-year-old man with bilateral plantar fibromatosis confirmed by ultrasound and MRI who underwent a wide excision followed by physical therapy and orthotic support with satisfactory evolution.
\end{abstract}

\section{Keywords}

Ledderhose's disease, Plantar fibromatosis, Plantar aponeurosis, Plantar fascia, Ultrasonography, MRI

\section{Introduction}

Ledderhose's disease or plantar fibromatosis is benign condition characterized by nodular fibroblastic proliferation of the plantar fascia. Diagnosis is based on clinical examination and may be confirmed by ultrasonography and MRI. Treatment is above all conservative and surgical management is reserved when conservative measures fail.

\section{Case Report}

A 46-year-old man with no significant medical or family history was referred for evaluation of bilateral nodules on the soles of both feet. The nodules had been present for more than 4 years but were gradually enlarging and the patient felt discomfort while walking.
On physical examination, he had subcutaneous nodules on the medial aspect of the plantar surface of the feet, more voluminous on the right. The patient was otherwise healthy and vital signs and laboratory tests were unremarkable.

Radiographs demonstrated absence of underlining bone disease. An ultrasound was performed and revealed bilateral hypoechoic nodules located in the distal part of the central bundle of the plantar fascia, which presented slight color Doppler flow. On MRI the nodules were iso to hypointense on T1 and T2-weighted images owing to their fibrous nature, hyperintense on STIR images and showed enhancement after contrast injection (Figure 1).

The diagnosis of Ledderhose disease was suspected based on the clinical-radiographic data. Owing to the progression of the disease and functional compromise, a wide excision with a safe margin was performed bilaterally. The diagnosis was confirmed by histopathology, which showed proliferation of fibrous tissue without associated malignancy. It was followed by post-operative physical therapy and orthotic support and had satisfactory evolution with no recurrence for at least four years follow-up.

\section{Discussion}

Ledderhose's disease is a rare, benign condition of unknown origin. It is a hyperproliferative disorder characterized by nodular fibrosis of the plantar fascia. It may occur at any age, with the greatest prevalence at

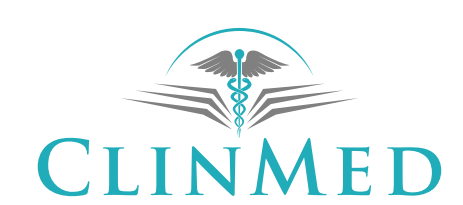

INTERNATIONAL LIBRARY
Citation: Vieira F, Canella C, Costa F, Severo A, Marchiori E (2017) Plantar Fibromatosis: An Unusual Cause of Plantar Pain. Int J Sports Exerc Med 3:078. doi.org/10.23937/2469-5718/1510078 Received: May 05, 2017; Accepted: November 20, 2017; Published: November 22, 2017 Copyright: (c) 2017 Vieira F, et al. This is an open-access article distributed under the terms of the Creative Commons Attribution License, which permits unrestricted use, distribution, and reproduction in any medium, provided the original author and source are credited. 

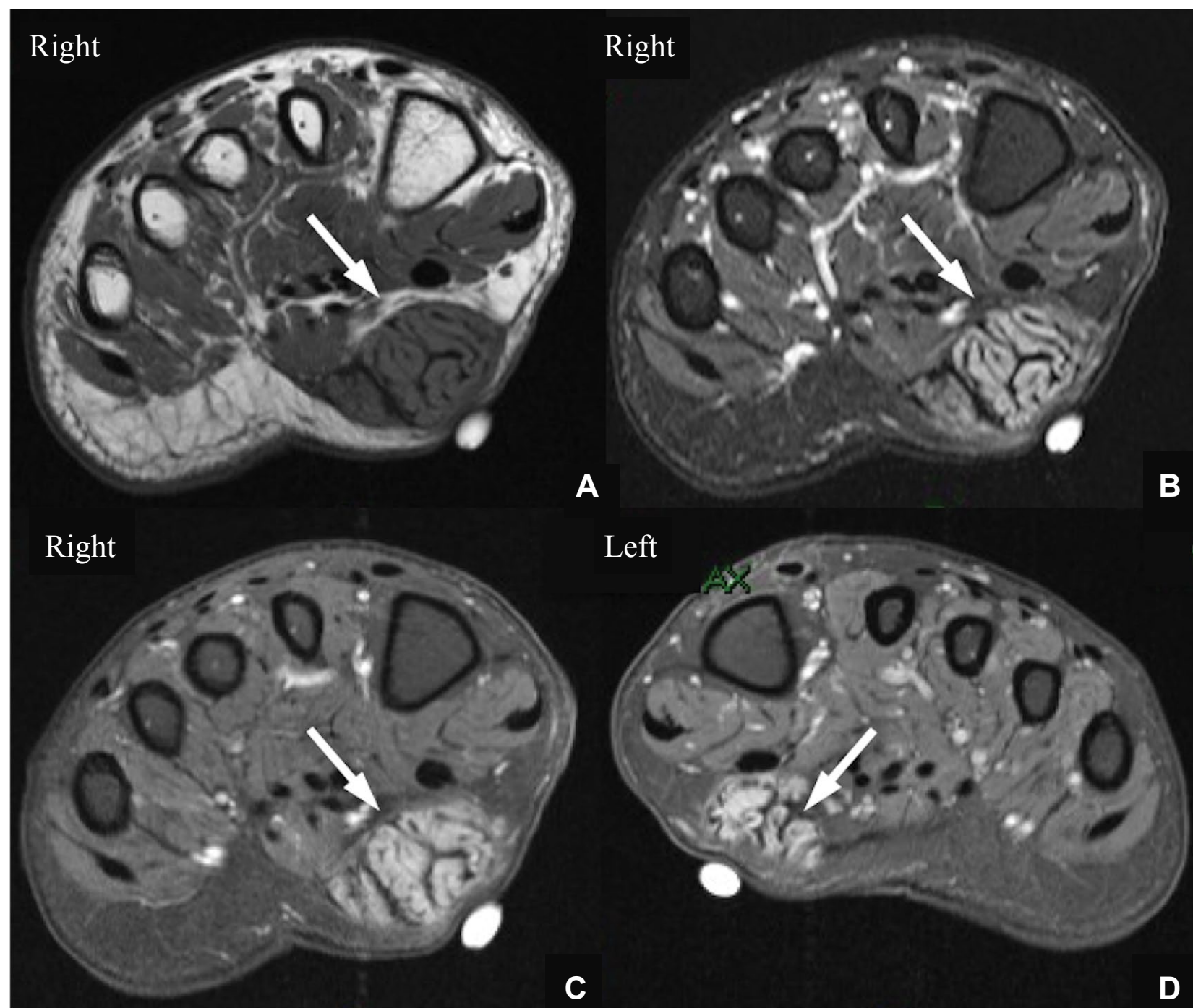

Figure 1: MRI axial images displays nodular thickening of the right distal plantar fascia (arrows), which is isointense to skeletal muscle on T1-WI (A) and hyperintense on STIR (B). T1-weighted, fat-suppressed images after contrast injection (C) show enhancement of the lesion. Note the bilateral involvement (D).

middle age and beyond and men are affected twice as often as women. It tends to involve the distal two thirds of the plantar fascia, usually superficially in its central bundle. Nodular lesions may be multiple and there is bilateral involvement in $25 \%$ of cases [1,2].

It may occur individually or in association with other fibromatoses such as palmar fibromatosis (Dupuytren's disease) and penile fibromatosis (La Peyronie's disease). An increased incidence of the disease has been reported with diabetes mellitus, alcohol addiction and epilepsy [2].

Clinically, this disease appears as one or more slowgrowing nodules generally localised in the medial portion of the sole, which become symptomatic when they increase in size, leading to walking disability. Unlike the effect of palmar lesions on the fingers, plantar lesions rarely result in flexion of the toes.

Diagnosis is above all clinical but some imaging modalities such as ultrasound and MRI can be useful to confirm the diagnosis and define the degree of extension of the disease. Histological analysis is reserved for doubtful cases to rule out malignancies.
The sonographic presentation of plantar fibroma is typically a fusiform hypoechoic lesion located superficially in the plantar aponeurosis [3]. The common MRI appearance is a nodule of low signal intensity on both T1- and T2-weighted sequences due to its fibrous nature and may show high signal on fluid-sensitive sequences. Following gadolinium injection there is considerable enhancement [4].

The most important differential diagnosis is nodular fasciitis and fibrosarcoma.

The choice of treatment depends on the degree of the pathological alterations and individual symptoms. Conservative treatment includes anti-inflammatory drugs, intralesional corticosteroid injections, physical therapy, and orthopedic insoles. Radiotherapy and extracorporeal shock wave therapy are also being used [5]. The surgical options are chosen when conservative measures fail to improve symptoms and prevent the progression of the disease. Surgery should relieve pain as well as maintain the patient's ability to walk. Local excision, wide excision, and complete fasciectomy are possible operative methods, however the recurrences rates are high [5]. 


\section{Conclusion}

Plantar fibromatosis is a rare benign condition characterized by local proliferation of fibrous nodular tissue within the plantar aponeurosis. The diagnosis of this disease is based on clinical examination but sonography and magnetic resonance imaging are useful to confirm the diagnosis.

Because the cause of Ledderhose disease is still unknown, numerous therapeutic approaches for its treatment are available, from conservative to surgical.

\section{Ethical Statements}

The authors declared no conflicts of interest and received no financial support with respect to the research, authorship, and/or publication of this article.

All authors actively participated in the preparation of the paper.
The work is original and is not in the process of being evaluated by any other scientific journal.

\section{References}

1. Omor Y, Dhaene B, Grijseels S, Alard S (2015) Ledderhose Disease: Clinical, Radiological (Ultrasound and MRI), and Anatomopathological Findings. Case Rep Orthop 2015: 741461.

2. Adib O, Noizet E, Croue A, Aubé C (2014) Ledderhose's disease: Radiologic/pathologic correlation of superficial plantar fibromatosis. Diagn Interv Imaging 95: 893-896.

3. Draghi F, Gitto S, Bortolotto C, Draghi AG, Belometti G (2017) Imaging of plantar fascia disorders: findings on plain radiography, ultrasound and magnetic resonance imaging. Insights Imaging 8: 69-78.

4. Watson-Ramirez L, Rasmussen SE, Warschaw KE, Mulloy JP, Elston DM (2001) Plantar fibromatosis: use of magnetic resonance imaging in diagnosis. Cutis 68: 219-222.

5. Veith NT, Tschernig T, Histing T, Madry H (2013) Plantar fibromatosis--topical review. Foot Ankle Int 34: 1742-1746. 